\title{
Caídas en el adulto mayor y factores de riesgo
}

\author{
Gabriel Gerardo Suárez, Víctor Manuel Velasco, María de Lourdes Limones, \\ Hugo Reyes, y Viviana Elizabeth Delgado \\ Universidad Autónoma de Coahuila (México)
}

El artículo presenta los resultados de una investigación respecto a la asociación de caídas con diferentes variables que determinan de manera significativa caídas en el adulto mayor. los factores de riesgo que se asocian a una caída en el adulto mayor son múltiples y generalmente existen más de uno, que tienen que ver con el estado de salud, funcionalidad y las condiciones del medio ambiente, así como a la interacción entre los distintos factores, particularmente; hipertensión, traumatismo, edad, y uso de bastón, riesgos que de consumarse condicionan categóricamente la autonomía, e independencia del adulto mayor, en la operatividad funcional de las actividades básica e instrumentales de la vida diaria, disminuyendo su calidad de vida y consecuentemente un envejecimiento saludable y exitoso. Es un estudio transversal en población abierta, se incluyeron a adultos mayores de 60 años de edad. Se realizó una entrevista, previo consentimiento informado para indagar datos sociodemográficos, antecedentes médicos, se aplicaron instrumentos para medir factores de riesgo; escala de factores predictivos de caídas, y dependencia, (KATZ). En los resultados obtenidos se encontró como factores predisponentes más frecuentes; edad avanzada, tener alguna enfermedad, diabetes o hipertensión, dificultad para su cuidado, utilizar aditamentos de apoyo para moverse, y la polifarmacia.

Palabras clave: Adulto, mayor, calidad de vida, caída, factores de riesgo.

Risk Factors and Falls in the Elderly. The article presents the results of an investigation regarding the association of falls with different variables that significantly determine falls in the elderly. The risk factors associated with a fall in the elderly are multiple and generally there are more than one. These factors have to do with the state of health, functionality and environmental conditions, as well as the interaction between the different factors, particularly; hypertension, trauma, age, and use of the cane, risks that are consummated categorically condition the autonomy and independence of the elderly in the functional operation of the basic and instrumental activities of daily life, reducing their quality of life and consequently a healthy and successful aging. This is a cross-sectional study in the open population, in which 60-year-old adults were included. With prior consent, an interview was conducted to investigate socio-demographic data and medical history, and instruments were applied to measure risk factors, scale of predictive factors of falls, and dependence (KATZ). In the results obtained, it was found as the most frequent predisposing factors; advanced age, diabetes, hypertension, or having any disease, as well as difficulty in caring, use supportive devices to move around, and poly-pharmacy.

Keywords: Adult, elderly, quality of life, fall, risk factors.

\footnotetext{
Correspondencia: Gabriel Gerardo Suárez Alemán. Universidad Autónoma de Coahuila, Escuela de Licenciatura en Enfermería Unidad Torreón. Monte Vesubio, s/n. C.P.: 27298. Torreón, Coahuila (México). E-mail: suag71@ @otmail.com
} 
De acuerdo a la Organización Mundial de la Salud (OMS), se define caída como la precipitación de un sujeto al suelo contra su voluntad como consecuencia de diversas situaciones, esto son acontecimientos involuntarios que hacen perder el equilibrio y caer ya sea sobre el suelo u otra superficie firme que detenga el cuerpo. Las caídas en los adultos mayores son un problema de salud pública de alta frecuencia a nivel mundial, así, se ha reportado que México ocupa el segundo lugar en América en cuanto a caídas de personas mayores (Costello y Edelstein, 2008; Smith, Wade, Mounce, Wilson, y Parrish, 2010). Se reporta que de los adultos mayores que viven en la comunidad, aproximadamente el $30 \%$ sufre de al menos una caída al año, siendo un porcentaje mayor cuando viven en instituciones. El 50\% de los adultos mayores que se caen, requieren atención médica y hasta el 10\% llegan a sufrir una fractura (Gillespie et al., 2009).

Es conocido que existen diversos cambios biológicos con el envejecimiento que incrementan el riesgo de caer (Quintero, 2009), por lo que las caídas se han considerado un gran síndrome geriátrico que debe estar presente en la mente del personal del área de la salud, por ser un problema frecuente y recurrente. Dentro de los múltiples factores asociados a una caída se encuentran por ejemplo la edad, la pérdida de reflejos, que facilita la pérdida de equilibrio (Millán, 2011). Los sujetos que presentan alteraciones en equilibrio presentan una prevalencia de caídas hasta $95 \%$ mayor que los que no tienen falta de equilibrio (Brito, Coqueiro, Fernandes, y de Jesús, 2014). De la misma forma, los adultos mayores que tienen alteraciones en su marcha incrementan la susceptibilidad de caer (Russell, Hill, Blackberry, Day, y Dharmage, 2006).

Otros factores asociados con las caídas son el sexo y el estado civil, así, se ha reportado que las mujeres tienen más riesgo de caerse que los hombres y que las personas casadas que viven con su pareja, se caen con menor frecuencia que las personas que viven solos por estar viudos, divorciados o solteros. También se han asociado a un aumento de las caídas, diversas enfermedades como la artritis, diabetes, depresión, incontinencia urinaria, sobrepeso (Bloch et al., 2010) y otras enfermedades crónicas y degenerativas que pueden asociarse con alteraciones del estado cognitivo (memoria, aprendizaje, inteligencia) que coadyuvan a la posibilidad de caerse, además del impacto psicosocial (Potter y Perry, 2008).

Enfermedades en órganos de los sentidos como el oído (otitis, otosclerosis), la visión (cataratas, glaucoma) y alteraciones de la musculatura cervical (artrosis), también se han asociado a incremento en posibilidad de caídas (Soares, 2010), así como la falta de ejercicio de manera sistemática y regular (Millán, 2011). Los problemas de los pies es otro factor común y frecuente que pueden predisponer a caídas por lo que es un punto importante a cuidar (Castro, 2005).

Las consecuencias de las caídas son múltiples e involucran el aspecto físico, psicológico, familiar, social, económico y del sistema de salud, ya que provoca incremento en la morbilidad y mortalidad en este grupo etario y tiene repercusiones sociales y 
psicológicas. Dentro de la esfera física, además del traumatismo, una caída puede producir contusiones, heridas, luxaciones y fracturas, principalmente en cadera, columna y extremidades que pueden llevar al ingreso al hospital, inmovilidad, discapacidad temporal o permanente y hasta la muerte.

En la esfera psicológica es frecuente encontrar el sentimiento de minusvalía, miedo de caer nuevamente, con la consecuente disminución de actividades habituales y con ello la pérdida de la independencia lo que aumenta aún más la morbilidad y mortalidad (Kamel, Abdulmajeed, e Ismael, 2013).

En la esfera familiar, cuando un adulto mayor se cae, puede volverse dependiente, requiriendo y demandando una mayor atención por parte de los familiares (Ory et al., 2010). En la esfera social, económica y de los sistemas de salud, al existir pérdida de la autonomía o discapacidad funcional importante, necesitará de periodos de rehabilitación prolongados con el consecuente incremento en los costos derivados de una atención médica a largo plazo y sobrecarga en los sistemas de salud pública (Bloch et al., 2010; Costello y Edelstein, 2008; Persad, Cook, y Giordani, 2010).

Existen muchas estrategias y acciones que pueden ayudar a disminuir la frecuencia y el impacto de las caídas en el adulto mayor, como programas de ejercicio supervisado por profesionales, medidas que permitan fortalecer la masa muscular, corrección de problemas visuales, eliminación de obstáculos, desarrollo de actividades dentro de una rutina o patrón, identificación de factores de riesgo mediante apoyos electrónicos, etc. (Bloch et al., 2010; Johnston, Grimmer-Somers, y Sutherland, 2010; Ory et al., 2010).

En estas acciones, es de suma importancia la participación de los profesionales de enfermería, ya que la enfermera tiene la facilidad de interactuar con los sujetos de edad avanzada y su familia en sus distintos medios (en casa, en instituciones, en su sitio laboral, etc.), para tener los elementos necesarios que le permitan evaluar de manera íntegra los distintos factores de riesgo de caer a los que se encuentra expuesto el adulto mayor, conocer los factores intrínsecos del sujeto, como los extrínsecos del medio, y con ello plantear estrategias de prevención y control específicos y personalizados para cada sujeto, por ejemplo la necesidad de cambios de estilos de vida, intervenciones para el fortalecimiento de masa muscular, prevención de osteoporosis, mejoramiento de movilidad articular, mejoramiento de equilibrio, uso de ropa y calzado adecuado, uso adecuado de bastón u otros equipos de apoyo, mejoramiento de la visión, vigilancia de un peso ideal, educación sobre la realización de prácticas seguras y limitación de las inseguras (subir a una silla o a una escalera), necesidad de apoyos en casa como barandales o agarraderas de apoyo, condiciones de iluminación y de orden adecuados (González, López, y Trujillo, 2005).

El fundamento para todas estas acciones, parte de un principio básico que Orem (1983), denomina autocuidado, que se refiere a la necesidad de que el individuo realice en 
forma habitual, voluntaria y consciente, las acciones y actividades que le permitan mantener la vida, la salud y su bienestar, de manera que el mismo sujeto sea su propio cuidador. Esto es un reto para el profesional de Enfermería, ya que como se mencionó, es precisamente este profesional de la salud quien tiene el contacto más directo con el sujeto y la familia en sus distintos medios, y debe buscar que el sujeto adulto mayor tome posturas activas frente al cuidado de su salud, de manera que a través de la información de los riesgos existentes para tener una caída, y el conocimiento de la manera de prevenirlos, sea el mismo sujeto quien tome las riendas de sus decisiones sobre lo que debe y puede hacer, y lo que debe y puede evitar, y que mediante las modificaciones positivas de hábitos, actitudes y costumbres (Tobón, 2003), disminuya la frecuencia y las consecuencias de las caídas (Diez, 2003).

El objetivo de este estudio es determinar las variables frecuentes que se asocian con caídas en el adulto mayor, mismas que debemos atender de manera preventiva para conservar y mantener la funcionalidad, independencia y bienestar, elementos básicos para promover una buena calidad de vida en la persona mayor, y un envejecimiento exitoso.

\section{MÉTODO}

\section{Participantes}

Estudio transversal en población abierta, 94 adultos mayores de 60 años de edad, seleccionados aleatoriamente en población abierta. Se incluyeron mayores de 60 años que aceptaron participar, se excluyen los que tuvieron puntuación del Mini-Mental State Examination (MMSE) menor de 12 y se eliminaron los que no completaron la recolección de datos.

\section{Instrumentos}

Entrevista previo consentimiento informado para indagar datos sociodemográficos, escolaridad, riesgo de caídas (escala de factores predictivos de caídas), y dependencia (Katz).

En la entrevista se obtuvo información sobre las siguientes variables: A) caídas, definiendo la caída como la consecuencia de cualquier acontecimiento que precipita al paciente al suelo en contra de su voluntad. Esta precipitación suele ser repentina e involuntaria. Puede ser referida por el paciente o por un testigo. B) Variables generales como edad en años cumplidos, sexo (hombre o mujer), educación (primaria, secundaria, estudio técnico, preparatoria o profesional). C) Variables de naturaleza social y familiar como la percepción de aceptación, aislamiento o agresión física, psicológica o social, vivir solo o con pareja estable, sitio de vivienda, vivir con cónyuge, hijos u otros familiares o amigos. D). Variables que tienen que ver con los aspectos económicos y laborales como 
sentirse útil, dependencia económica, actividad laboral. E) Variables que tienen que ver con la capacidad física y la salud como la presencia de enfermedades, discapacidad, dependencia física, depresión, polifarmacia, y F) Variables que tienen que ver con los estilos de vida como el tabaquismo, uso de alcohol, alimentación, actividades de recreación.

Para la obtención de la información se elaboró una entrevista estructurada que incluyera los aspectos mencionados, que se validó mediante consenso de expertos, validación de contenido y que obtuvo un alfa de Cronbach de 0.85 de consistencia interna. Las entrevistas se aplicaron por tres entrevistadores previamente adiestrados para tal fin, obteniendo un coeficiente kappa de 0.8. Además, se aplicaron las siguientes escalas: Escala de factores predictivos de caídas, la cual contempla cuatro ítems; antecedentes de caídas en los últimos cuatro meses, problemas visuales, incontinencia urinaria, y limitación funcional, la respuesta positiva a cada una de ellas, determina categóricamente riesgo de caídas cuyo puntaje máximo es de 15 puntos. Katz, validada en español (consistencia interna entre 0.84 a 0.96 de alfa de Cronbach, correlación interna de Spearman de 0.94 y concordancia interobservador de 0.8 (kappa). Se trata de un instrumento para evaluar la independencia de un enfermo en cuanto a la realización de las actividades básicas de la vida diaria. En muchos casos, puede ser contestado por el propio enfermo, en otros deben ser los familiares, y en todo caso, deberá mediar una exploración adecuada. Se trata de seis ítems dicotómicos que evalúan la capacidad o incapacidad de efectuar una serie de tareas. La capacidad para realizar cada una de las tareas se valora con 0 , mientras que la incapacidad, con 1. El programa evalúa la puntuación final, y clasifica al enfermo en tres grados de incapacidad. Esta suele ser la valoración más utilizada, aunque originariamente los autores agrupaban progresivamente cada uno de los ítems, señalando con diversas letras (A, B, C...etc.) el tipo de dependencia, según la función estudiada fuera clasificada de una forma u otra.

\section{Análisis estadístico}

Análisis en SPSS 20.0 para determinar asociación de caídas, con sexo, dependencia, deterioro cognitivo, presencia de diabetes mellitus, hipertensión, tener dificultad en su cuidado, uso de aditamentos para apoyo, necesidad de aditamentos auditivos o visuales (anteojos), traumatismos, polifarmacia o tener más de 70 años.

\section{RESULTADOS}

Se estudiaron 94 adultos mayores, con una edad media de $70.4 \pm 7.57$ años, de los cuales el 54\% (51) son mujeres, 46\% (43) son hombres. Un 72\% (68 sujetos) refirieron alguna caída en el último año. 
Los factores predisponentes para caerse que se encontraron más frecuentes fueron tener alguna enfermedad como diabetes o hipertensión, dificultad para su cuidado, Utilizar aditamentos de apoyo para moverse y la polifarmacia (tabla 1), aunque los únicos que se asociaron significativamente fueron tener hipertensión, traumatismo, sitio de caídas, edad > 70 años (tabla 2). Se caen más los que utilizan bastón (25\%) que los que no lo utilizan $(7.7 \%$ ), aunque la diferencia no fue significativa (tabla 3). No se encontró asociación entre caídas y el sexo, el uso de otros aditamentos de apoyo para moverse o auxiliares auditivos o uso de anteojos, presencia de otras enfermedades y el resto de variables estudiadas.

De acuerdo con la escala de riesgo de caídas, 54 sujetos se encontraban con riesgo de caídas, y se cayeron en el último año 68 sujetos (Tabla 4), lo que le confiere un valor de Sensibilidad 69.12 (IC 95\% de 68.32-69.92), especificidad 73.08 (IC 95\% de 71.05-75.10), VPP 87 (IC 95\% de 86-88), VPN 47.5 (IC 95\% de 46.16-48.84).

Tabla 1. Características generales de la muestra

\begin{tabular}{lcc}
\hline SI & & NO \\
\hline Dificultad para sucuidado & 56 & 44 \\
\hline SuficienteAyuda & 79.5 & 20.5 \\
\hline Necesita adaptación encasa & 48.4 & 51.6 \\
\hline TieneAdaptación & 19,6 & 80.4 \\
\hline Tiene algunaenfermedad & 80 & 14 \\
\hline $\begin{array}{l}\text { Utiliza algún aditamento de apoyo para } \\
\text { moverse (Bastón, muletas, Andadera, Silla } \\
\text { deRuedas) }\end{array}$ & 55 & 38 \\
\hline Utilizaanteojos & 36 & 58 \\
\hline Utiliza auxiliarauditivo & 4 & 90 \\
\hline Edad arriba de 70años & 34 & 60 \\
\hline DependenciaFísica & 39 & 54 \\
\hline Polifarmacia & 55 & 31 \\
\hline Hipertensiónarterial & 68 & 26 \\
\hline Mayores 70años & 34 & 68 \\
\hline
\end{tabular}

Tabla 2. Asociación de caídas con diferentes variables

\begin{tabular}{|c|c|c|c|c|c|}
\hline & & Caída & No caída & OR (IC95\%) & $p$ \\
\hline \multirow{2}{*}{ Hipertensión } & $\mathrm{Si}$ & 53 & 15 & \multirow{2}{*}{$1.92(1.01-3.61)$} & \multirow{2}{*}{0.05} \\
\hline & No & 15 & 11 & & \\
\hline \multirow{2}{*}{ Traumatismo } & $\mathrm{Si}$ & 19 & 2 & \multirow{2}{*}{$4.75(1.02-22.09)$} & \multirow{2}{*}{0.032} \\
\hline & No & 48 & 24 & & \\
\hline \multirow{2}{*}{ Edad } & $<70$ & 39 & 21 & \multirow{2}{*}{$3.12(1.07-9,26)$} & \multirow{2}{*}{0.035} \\
\hline & 71 ó > & 29 & 5 & & \\
\hline \multirow{2}{*}{ Sitio de caída } & Casa & 55 & 0 & \multirow{2}{*}{$5.321(3.28-8.52)$} & \multirow{2}{*}{0.000} \\
\hline & Fuera & 13 & 26 & & \\
\hline
\end{tabular}

Tabla 3. Asociación de caídas con el uso de bastón

\begin{tabular}{lcccc}
\hline & Caída & No caída & OR & p \\
\hline Uso de bastón & 24 & 2 & & \multirow{2}{*}{$4.00(0.86-18.72)$} \\
\cline { 1 - 3 } No usa bastón & 51 & 17 & & 0.062 \\
\hline
\end{tabular}


Tabla 4. Sensibilidad y especificidad de Escala de riesgo de caídas

\begin{tabular}{|c|c|c|c|c|c|c|}
\hline \multicolumn{4}{|c|}{ Caídas } & \multirow{2}{*}{ Total } & Sensibilidad & Especificidad \\
\hline & \multicolumn{2}{|c|}{ No Caída } & Caída & & & \\
\hline \multirow{3}{*}{$\begin{array}{l}\text { Riesgo } \\
\text { Caída }\end{array}$} & Sin Riesgo & 19 & 21 & 40 & 69.12 & 73.08 \\
\hline & Riesgo de caída & 7 & 47 & 54 & $\begin{array}{c}\text { IC } 95 \% 68.32- \\
69.92 \\
\end{array}$ & $\begin{array}{c}\text { IC 95\% } 71.05- \\
75.10 \\
\end{array}$ \\
\hline & Total & 26 & 68 & 94 & & \\
\hline
\end{tabular}

\section{CONCLUSIONES/DISCUSIÓN}

En el presente estudio encontramos un $72 \%$ de sujetos que refirieron alguna caída en el último año, lo que es superior en relación a lo reportado por otros autores (Gillespie et al., 2009), que reportan que de las personas adultos mayores que viven en la comunidad, alrededor del $30 \%$ sufren de al menos una caída al año. Otros estudios en México reportan prevalencia de caídas de 43\% (Barrantes et al., 2007; Leiva et al., 2015), mientras en otros países de Sudamérica se reportan entre 29\% y 34\% (Reyes et al., 2005).

Estudios como el de Leiva et al. (2017), en Chile reportan prevalencias hasta 73.4\%. Las diferencias tienen que ver con el tipo de población estudiada, ya que nuestro estudio, aunque fue en población abierta, la base de datos de adultos mayores para su localización partió de población adulta mayor que había estado hospitalizada en los últimos seis meses, al igual que la población de Chile, que se efectuó solo en sujetos que utilizaban bastón como parte de un programa de entrenamiento.

Los factores que se asocian a una caída en el adulto mayor son múltiples y generalmente existen más de uno, que tienen que ver con el estado de salud, competencia y funcionalidad del adulto mayor, sus habilidades y las condiciones del medio ambiente, tanto dentro como fuera de casa (Leiva et al., 2015), así como a la interacción entre los distintos factores (Hill et al., 2009). En nuestro estudio encontramos asociación solamente con algunos de ellos, como el tener hipertensión arterial, situación reportada por Millán (2011). Debemos tener presente que, a mayor edad, la prevalencia de hipertensión es mayor, además el paciente con hipertensión es más susceptible de alteraciones del flujo sanguíneo cerebral que puedan generar un cuadro sincopal, además del efecto secundario de los antihipertensivos, lo que pudiera explicar su asociación. En el estudio de Machado et al. (2014) el $74.3 \%$ de los adultos mayores que tuvieron caídas, tenían hipertensión arterial, similar a nuestro estudio en el que $77.9 \%$ de los sujetos con hipertensión tuvieron caídas versus el $57 \%$ de los que no tuvieron caídas.

De los adultos mayores que sufrieron una caída, el $42 \%$ tenían más de 70 años, en comparación con los que no sufrieron caídas de los que el 19.2\% eran mayores de 70 años. Un estudio en Chile (Leiva et al., 2017) reportan que la media de edad de quienes presentaron caídas fue de 76 años mientras que otro estudio en México refiere una media de edad de 78 años (Leiva et al., 2015). Esta situación es explicable por el deterioro osteomuscular, y neurológico que se acentúa con el tiempo. 
Referente al sitio de caída, el 80.9\% se cayó dentro del domicilio. Esta situación ha sido descrita en otros estudios, por ejemplo, Santillana (2002) en México refiere que el $62 \%$ de las caídas en mujeres ocurren en casa. Fhon et al. (2012) en Brasil también refieren que el sitio más frecuente de caída fue el interior de la casa (66.6\%) principalmente en el baño, el patio y el dormitorio. En España, Formiga et al. (2006) reporta un 68\%, y Castro (2005) refiere el $80 \%$ de caídas en domicilio, sin embargo, existe un estudio efectuado en el Centro de Rehabilitación y Educación Especial (CREE), en la Ciudad de Campeche, México, que reporta que solamente el $26.7 \%$ de las caídas ocurrieron en el hogar y el 73.3\% ocurrieron en la vía pública (Guerrero-Ceh et al., 2016). Las diferencias encontradas muy probablemente tangan que ver con la forma de seleccionar la población estudiada, el sitio de la entrevista y el punto de origen de la misma.

En el presente estudio se aprecia que de las personas que tuvieron caídas, el $25 \%$ utilizaban bastón, mientras que de los que no tuvieron caídas solo el $7.7 \%$ lo utilizaban. Esta situación en apariencia es contradictoria, ya que el bastón es un medio de apoyo, un dispositivo de ayuda para caminar, por lo que esperaríamos menos caídas en quienes utilizan el bastón, sin embargo, depende mucho de las condiciones de su uso, por ejemplo, en un estudio en Chile, entre personas que se les adiestró en el uso del bastón, solo el 7,8\% refirió que utiliza el bastón cuando se moviliza dentro de la casa, el 45,3\% cuando se moviliza fuera de la casa, el $46,9 \%$ cuando se moviliza por todas partes; y el 48,4\% expresó que no le agrada utilizar el bastón (Leiva et al., 2017), lo cual deja en relevancia que si bien es un elemento de apoyo para el adulto mayor, también les recuerda constantemente sus limitaciones y su uso tiene interacciones complejas entre el individuo y sus limitaciones, el medio ambiente y la actividad que desarrolle el individuo, por lo que el bastón se ha considerado de hecho un factor de riesgo para las caídas (Iwarsson et al., 2009), por lo que no basta con utilizar un bastón de apoyo, sino que es necesario evaluar su altura, mano en que se utiliza, postura corporal ya que los errores en cuanto a su uso puede conducir a no lograr el objetivo por el cual se indicó y a incrementar el riesgo de caer o el número de caídas (Liu et al., 2011).

La escala de riesgo de caídas utilizada nos mostró valores de Sensibilidad y especificidad útiles, al igual que los valores predictivos positivos (VPP), y los valores predictivos negativos (VPN).

No se encontró asociación entre caídas y el sexo, ni con en el uso de otros aditamentos de apoyo para moverse o auxiliares auditivos o uso de anteojos, presencia de otras enfermedades y el resto de variables estudiadas.

El riesgo de caídas sigue siendo una constante en los adultos mayores (González et al., 2005), por lo que es importante implementar programas de salud que vayan orientados a disminuir las tasas de caídas de los adultos mayores, en las comunidades, hospitales, asilos, y en el hogar, coadyuvando con esto, a disminuir las 
complicaciones que trae consigo las caídas, y evitar entre otras cosas, el síndrome de inmovilidad.

Los factores asociados a caídas en el adulto mayor son de índole multifactorial, en el presente estudio se midieron solo algunas de la cuales resultaron ser significativas como las variables; edad, caídas más en casa o fuera de ella, enfermedades como la hipertensión arterial, polifarmacia, y la falta de adecuaciones en casa, las cuales constituyen factores de riesgo categóricos para la consumación de las caídas en el adulto mayor, por lo que se plantea como objetivo general considerar estas y otras variables de riesgo de caídas, abordándolas desde un contexto y entorno preventivo, que limiten o minimicen el riesgo de eventos adversos que se traduzcan en perdida de la funcionalidad y por ende en la autonomía del adulto mayor, en sus actividades de la vida diaria.

\section{REFERENCIAS}

Barrantes, M., García, E.J., Gutiérrez, L.M., y Miguel, A. (2007). Dependencia funcional y enfermedades crónicas en ancianos mexicanos. Salud Pública de México, 49(4), 459- 466.

Bloch, F., Thibaud, B., Dugué, M., Brèque, C., Rigaud, A.S., y Kemoun, G. (2010). Episodes of falling among elderly people. A systematic review and meta-analysis of social and demographic predisposing characteristics. Clinics, 65(9), 895-903.

Brito, T.A., Coqueiro, R.S., Fernandes, M.H., and de Jesús C.S. (2014). Determinan ts of falls in Community-dwelling elderly: Hierarchical analysis. Public Health Nursing, 31(4), 290-297.

Castro, V. (2005). Intervención gerontológica en prevención de caídas. Herder: Millan.

Costello, E., y Edelstein, J.E. (2008). Update on falls prevention for community-dwelling older adults. Review of Single and Multifactorial Intervention Programs, 45(8), 1135-1152.

Fhon, J.R., Fabrício-Wehbe, S.C., Vendruscolo, T.R., Stackfleth, R., Marques, S., y Rodrigues, R.A. (2012). Caídas en el adulto mayor y su relación con la capacidad functional. Revista Latino-Americana Enfermagem, 20(5), 28-45.

Formiga, F., Ruiz, D., López-Soto, A., Duaso, E., Chivite, D., y Pérez, J.M. (2006). Circunstancias asociadas a las caídas responsables de fractura de fémur en pacientes ancianos. Diferencias por grupo de edad y género. Revista Clínica Española, 7(3), 314-318.

Gillespie, L.D., Gillespie, W.J., Robertson, M.C., Lamb, S.E., Cumming, R.G., y Withdrawn, B.H. (2009). Interventions for preventing falls in elderly people. Cochrane Database of Systematic Reviews, 15(2), 5.

González, C.B., López, R.V.M., y Trujillo, S.Z. (2005). Guía de práctica clínica para la prevención de caídas en el adulto mayor. Revista Médica del IMSS, 43(5), 425-441.

Guerrero-Ceh, J.G., Sarabia-Alcocer, B., y Can-Valle, A.R. (2016). Incidencia del síndrome de caídas en el hogar. Estudio realizado en personas mayores en el rango de edad de 60 a 80 años. RIDE: Revista Iberoamericana para la Investigación y el Desarrollo Educativo, 6(12), 46-59.

Hill, E.E., Nguyen, T.T., Shaha, M., Wenzel, J.J., De Forge, B.R., y Spellbring, A.M. (2009) Personenvironment interactions contributing to nursing home resident falls. Research in Gerontological Nursing, 2(4), 287-296.

Iwarsson, S., Horstmann, V., Carlsson, G., Oswald, F., y Wahl, H.W. (2009). Person-environment fit predicts falls in older adults better than the consideration of environmental hazards only. Clinical Rehabilitation, 23(6), 558-567. 
Johnston, K., Grimmer-Somers, K., y Sutherland, M. (2010). Perspectives on use of personal alarms by older fallers. International Journal of General Medicine, 3, 231-237.

Kamel, M.H., Abdulmajeed, A.A., e Ismael, S.E. (2013). Risk factors of falls a mong elderly living in urban Suez-Egypt. Pan African Medical Journal, 14, 1609.

Leiva, J., León, J., y Cortes, J. (2017) Uso de bastón y caídas en adultos mayores chilenos de la comunidad. Ciencia y Enfermería, 23(3), 11-21.

Leiva, J.A., Salazar, B.C., Gallegos, E.C., Gómez, M.V., y Hunter, K.F. (2015). Connection between competence, usability, environment and risk of falls in elderly adults. Revista Latino-Americana Enfermagem, 23(6), 1139-1148.

Diez, M.I. (2003). Manual de enfermería. España: Lexus Editores.

Liu, H.H., Eaves, J., Wang, W., Womack, J., y Bullock, P. (2011). Assessment of canes used by older adults in senior living communities. Archives of Gerontology and Geriatrics, 52(3), 299-303.

Machado, R.L., Bazan, M.A., e Izaguirre, M. (2014). Principales factores de riesgo asociados a caídas en ancianos del área de la salud. Medisan, 18(2), 156-161.

Millán, J. (2011). Gerontología y geriatría, valoración e intervención. Enfermería Clínica. Madrid, España: McGraw Hill.

Orem, D. (1983). Teoría del Déficit de Autocuidado. Modelos y Teorías en Enfermería. España: Harcourt-Brace.

Organización Mundial de la Salud (2003). Recuperado de: http://www.who.int/ media centre/factsheets/fs344/es/Ory.

Ory, M.G., Smith, M.L., Wade, A., Mounce, C., Wilson, A., y Parrish, R. (2010). Implementing and disseminatingan evidence-based program to prevent falls in older adults. Texas, 7(6), A130.

Persad, C.C., Cook, S., y Giordani, B. Assessing falls in theeld erly. (2010). Should we use simple screening testsor a comprehensive fall risk evaluation. European Journal of Physical and Rehabilitation Medicine, 46(2), 249-259.

Potter, P.A., y Perry, A.G. (2008). Valoración de las necesidades del anciano. Fundamentos de enfermería. Madrid España: McGraw-Hill.

Quintero, C.M.V. (2009). Riesgo de caídas en las personas mayores institucionalizadas en hogares geriátricos del distrito de Barranquilla. Revista Salud en Movimiento, 1(1), 21-23.

Reyes, C.A., Al-Snih, S., y Markides, K.S. (2005). Falls among elderly persons in Latin America and the Caribbean and among elderly Mexican-Americans. Revista Panamericana Salud Pública, 17(5-6), 362-369.

Russell, M.A., Hill, K.D., Blackberry, I., Day, L.L., y Dharmage, S.C. (2006). Falls risk and Functional decline in older fallers discharge directly from emergency departments. Journal of Gerontology Medical Sciences, 61(10), 1090-1095.

Soares, A.V. (2010). A contribuição visual para o controle postural. Revista de Neurociencia, 18(3), 370-379.

Tobón, O. (2003). El Autocuidado: Una Habilidad para Vivir. Revista Hacia la Promoción de la Salud, 8, 3-8.

Recibido: 19 de septiembre de 2019 Recepción Modificaciones: 03 de octubre de 2019

Aceptado: 14 de octubre de 2019 